Check for updates

Cite this: RSC Adv., 2017, 7, 26717

Accepted 10th May 2017

DOI: $10.1039 / \mathrm{c} 7 \mathrm{ra0} 4328 \mathrm{~g}$

rsc.li/rsc-advances

\title{
Interfacial defect engineering over fusiform bismuth vanadate photocatalyst enables to excellent solar-to-chemical energy coupling $\dagger$
}

\author{
Chunjing Shi, Xiaoli Dong, (D) * Jiawei Wang, Xiuying Wang, Hongchao Ma \\ and Xiufang Zhang
}

The presence of surface oxygen vacancies over oxide semiconductors plays a versatile role in the development of light-driven organic degradation and energy production. Simultaneously, the role of defect sites successfully forms an ideal model for the photocatalytic responsiveness over $\mathrm{BiVO}_{4}-\mathrm{OV}$ in the NIR region.

With the development of industrialization and rapid growth of the population, the global energy crisis and the serious problems accompanying combustion of fossil fuels threaten our environment, climate and human health. ${ }^{1}$ Therefore, we suggest that a simple and efficient strategy can effectively solve aforementioned problems, that is quite important but challenging. ${ }^{2}$ The sustainable and environmentally friendly lightdriven technologies have obtained considerable interdisciplinary attention in recent years. At present, metal oxide semiconductors as efficient catalysts for $\mathrm{H}_{2}$ production from water splitting and using solar light energy for the degradation of environmental pollutants have been investigated for various potential applications because of their high thermal conductivity, high chemical stability and low environmental toxicity. As previous report, the photocatalysts such as $\mathrm{MoS}_{2}, \mathrm{ZnSnO}_{3}, \mathrm{~g}$ $\mathrm{C}_{3} \mathrm{~N}_{4}$ and $3 \mathrm{C}-\mathrm{SiC},{ }^{3}$ were demonstrated profound improvement of its photocatalytic activity. They hold a great promise to realize the fast pollutant degradation and efficient energy generation via utilizing solar energy. However, sunlight basically consists of $44 \%$ visible light, $3 \%$ ultraviolet and the remainder infrared light. ${ }^{4}$ Unfortunately, the most of conventional photocatalysts only can utilize UV or/and visible light to achieve the solar-tochemical energy conversion. ${ }^{5}$ To overcome the grant challenge, we tried best to effectively broaden the optical window of conventional photocatalysts and endow them with considerable utilization efficiency of near infrared light. This kind of photocatalysts could realize the remarkable enhancement of photocatalytic activity. Besides, the main limitation of energy conversion originates from two bottlenecks. They are poor

School of Light Industry and Chemical Engineering, Dalian Polytechnic University, \#1 Qinggongyuan, Dalian 116034, P R China.E-mail:dongxl@dlpu.edu.cn

$\dagger$ Electronic supplementary information (ESI) available: Detailed experimental section, photocatalytic activity test of $\mathrm{BiVO}_{4}-\mathrm{OV}$ and $\mathrm{BiVO}_{4}-\mathrm{P}$. Raman spectra, high resolution $\mathrm{Bi}$ 4f and $\mathrm{V} 2 \mathrm{p}$ XPS spectra of $\mathrm{BiVO}_{4}-\mathrm{OV}$ and $\mathrm{BiVO}_{4}-\mathrm{P}, \mathrm{PL}$ spectra. See DOI: $10.1039 / \mathrm{c} 7 \mathrm{ra} 04328 \mathrm{~g}$ transition probability of photoexcited electrons to the conduction band under solar light illumination and serious photogenerated electron-hole recombination. In order to solve the problems, it is very important for the researchers to seek a desirable material that could improve fundamentally properties of the sunlight harvesting and the carrier separation/ transmission.

To date, only a few photocatalytic materials were reported that can efficiently utilize NIR light to achieve the pollutant decomposition and the clean energy production. In the regard, upconversion material doped photocatalyst, narrow band gap photocatalyst and plasmon-assisted photocatalyst, have been successfully presented to improve the photocatalytic activity. ${ }^{6}$ However, some photocatalytic materials still suffer from intrinsic drawbacks. For instance, the applications of narrowband gap photocatalysts are severely restricted owing to their inappropriate energy band positions lead to weak reduction or oxidation power. ${ }^{7}$ Hence, the conventional narrow band gap photocatalysts cannot efficiently realize solar-to-chemical energy conversion. On the other hand, modified photocatalysts with complicated component such as nitrogen, sulfide and noble metal possibly result in environmental deterioration due to poisonous substances enter into the ecological environment. On this occasion, it is important meaning to explore an appropriate candidate for designing efficient semiconductor.

Compared with the conventional and studied photocatalysts, oxygen vacancy defects (OVs) on the surface is the most common type of defects in oxide semiconductor. In particular, it provides perfect sites for enhanced $\mathrm{O}_{2}$ adsorption and activation. Meanwhile, OVs is a remarkable strategy to modify the band gap of semiconductors and adjust their electronic structures. In view of the point, it has been demonstrated that semiconductors surface with oxygen vacancy could efficaciously stretch photo-response from UV to visible light region. ${ }^{8}$ In this case, oxygen vacancy acts as the conduction band of semiconductor materials leading 
to optimizing electronic band structure, boosting electron-hole pair separation and suppressing the photoexcited electron-hole recombination in bulk materials. Thus, oxygen defects can make semiconductor materials enhance the ability of utilizing solar light. ${ }^{9}$ The result gives us an inspiration which semiconductor with OVs may be responsive to NIR light region. To validate this consideration, we selected bismuth vanadate $\left(\mathrm{BiVO}_{4}\right)$ as the credible photocatalyst. Bismuth vanadate $\left(\mathrm{BiVO}_{4}\right)$ is one of the most promising nano-materials, due to its low cost, chemical and thermal stability, and environmentally friendly properties. ${ }^{10}$ However, the widespread application of $\mathrm{BiVO}_{4}$ as an efficient photocatalyst is hindered by its poor light absorption and insufficient charge separation efficiency. In this study, we considered that OV-incorporated $\mathrm{BiVO}_{4}$ can be highly efficient degradation of pollutions under broad solar spectrum ranging from UV to NIR.

The desired $\mathrm{BiVO}_{4}$-OV was prepared by a conventional and facile one-step solvothermal method. During the process, glycerol acts as reducing agent. Based on redox reaction mechanism, the hydroxyl removed selectively oxygen atoms from the $\mathrm{BiVO}_{4}$ surface, resulting in the formation of oxygen vacancy. For comparison, the sample of $\mathrm{BiVO}_{4}$-OV was putted in an appropriate crucible. Then, it was annealed to $300{ }^{\circ} \mathrm{C}$ for $5 \mathrm{~h}$ in an airrich atmosphere muffle furnace. After cooling to room temperature, the prepared of the sample without oxygen vacancy was obtained and marked as $\mathrm{BiVO}_{4}-\mathrm{P}$. The detailed experimental procedure was provided in ESI.

The morphology of $\mathrm{BiVO}_{4}-\mathrm{OV}$ and $\mathrm{BiVO}_{4}-\mathrm{P}$ were charactered by field emission scanning electron microscopy (FESEM) and transmission electron microscopy (TEM). The picture of FESEM indicated uniform fusiform shape with relatively porous and unsmooth surface, and the morphology had no obvious change between $\mathrm{BiVO}_{4}-\mathrm{OV}$ and $\mathrm{BiVO}_{4}-\mathrm{P}$ in Fig. $1 \mathrm{a}$ and b. Moreover, as shown in Fig. 1c and d, transmission electron microscopy (TEM) was performed which demonstrates the high crystallinity nature of semblable $\mathrm{BiVO}_{4}$ and have their (040) facets exposed. In addition, it can be seen from the high-resolution TEM images of $\mathrm{BiVO}_{4} \mathrm{-OV}$ that shows the crystal lattice fringes and the distance of neighboring fringes was measured to be $0.295 \mathrm{~nm}$ (Fig. 1f), which accorded with the (040) planes of $\mathrm{BiVO}_{4}-\mathrm{OV}$. The lack of lattice was drawn with the dotted circle and a disordered layer of 1.59-1.77 nm thick could be obviously seen at the sample edges. The above appearance could reveal the surface damage due to oxygen atoms moved from the $\mathrm{BiVO}_{4}$ surface resulting in the formation of OVs (Fig. 1f). ${ }^{11}$ However, the edges of $\mathrm{BiVO}_{4}-\mathrm{P}$ are clearer lattice fringes. For compared with $\mathrm{BiVO}_{4}{ }^{-}$ $\mathrm{OV}, \mathrm{BiVO}_{4}-\mathrm{P}$ has no similar phenomenon (Fig. 1e).

To further affirm the presence of OVs, the as-synthetized $\mathrm{BiVO}_{4}-\mathrm{OV}$ and $\mathrm{BiVO}_{4}-\mathrm{P}$ were charactered by electron spin resonance (ESR), a tool for examining unpaired electrons in materials. The spectrogram of $\mathrm{BiVO}_{4}-\mathrm{OV}$ uniquely exhibits a symmetrical ESR signal at $g=2.002$, due to the electron trapping at oxygen vacancies (Fig. 2a). ${ }^{12}$ Therefore, the successful formation of OVs on $\mathrm{BiVO}_{4}$ surface via our facile onestep glycol-assisted solvothermal approach was verified. On the contrary, the ESR spectrum of the $\mathrm{BiVO}_{4}-\mathrm{P}$ could not emerge the corresponding signal. This phenomenon proves that the high
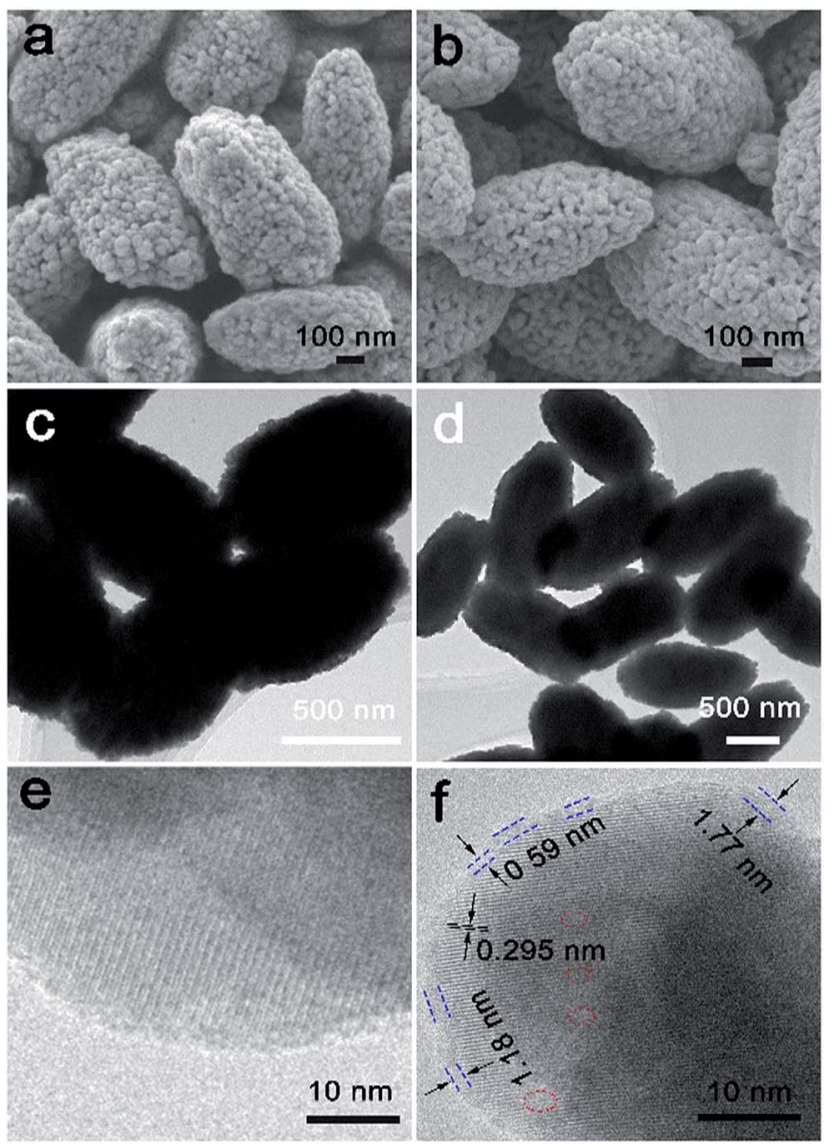

Fig. 1 (a) FESEM images of $\mathrm{BiVO}_{4}-\mathrm{P}$ and (b) $\mathrm{BiVO}_{4}-\mathrm{OV}$. (c) TEM images of $\mathrm{BiVO}_{4}-\mathrm{P}$ and (d) $\mathrm{BiVO}_{4}-\mathrm{OV}$. (e) HRTEM image of $\mathrm{BiVO}_{4}-\mathrm{P}$ and (f) $\mathrm{BiVO}_{4}-\mathrm{OV}$ (the lattice distortion and lack of the lattice).

temperature annealing process could eliminate all the OVs on $\mathrm{BiVO}_{4}$ surface.

XRD were employed to characterize the crystal phase of the sample $\mathrm{BiVO}_{4}-\mathrm{P}$ and $\mathrm{BiVO}_{4}-\mathrm{OV}$. The pattern was depicted in Fig. $2 \mathrm{~b}$, the $\mathrm{XRD}$ diffraction peaks of $\mathrm{BiVO}_{4}-\mathrm{P}$ are corresponding to the standard well-indexed to the phase of monoclinic $\mathrm{BiVO}_{4}$ (JCPDS card no. 14-0688), which disclose the obtained sample without any impurities. Nevertheless, the typical XRD diffraction character of $\mathrm{BiVO}_{4}$-OV had no the homologous crystal facet (251) and the intensity of diffraction peak is severely weaker than those of $\mathrm{BiVO}_{4}-\mathrm{P}$ in Fig. $2 \mathrm{~b} .{ }^{13}$ The crystallinity weakening are attributed to the displacement of oxygen atoms resulting in compressive stress state. Previous reports also showed the similar phenomenon of OVs-induced crystallinity weakening. ${ }^{14}$

Raman spectroscopy can provide structural information to witness the existence of oxygen vacancy. To avert possible ambiguities in the structural information of sample, characteristic Raman peaks spectrogram of the sample $\mathrm{BiVO}_{4}-\mathrm{OV}$ and $\mathrm{BiVO}_{4}-\mathrm{P}$ were performed by Raman spectroscopy used different Raman laser lines. Based on Fig. 2c, it can found that the external mode (rotation/translation) occurred at around 210 $\mathrm{cm}^{-1}$ and $123.8 \mathrm{~cm}^{-1}$. The most intense peak at $820.36 \mathrm{~cm}^{-1}$ and the weak shoulder at about $713 \mathrm{~cm}^{-1}$ are attributed to the stretching modes of the two vibrational modes of the $\mathrm{V}-\mathrm{O}$ 

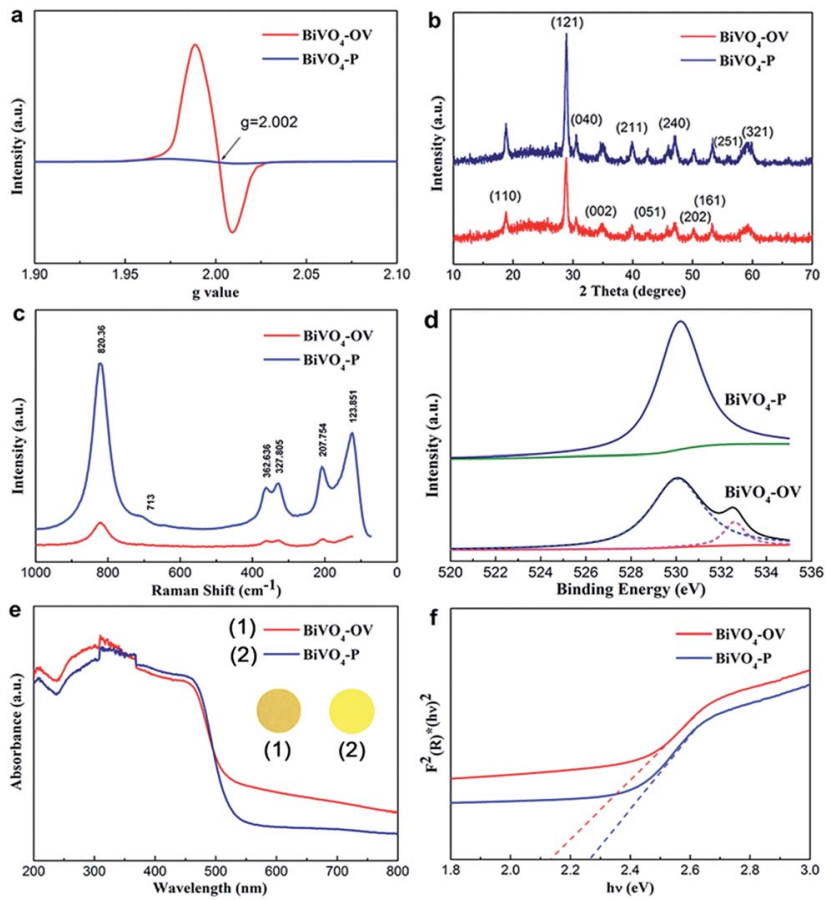

Fig. 2 (a) ESR spectra, (b) XRD pattern, (c) Raman spectra, (d) high resolution $\mathrm{O}$ 1s spectra, (e) UV/Vis absorption spectra (inset shows the colours of photocatalysts) and (f) Tauc plot of $\mathrm{BiVO}_{4}-\mathrm{P}$ and $\mathrm{BiVO}_{4}-\mathrm{OV}$.

bonds. ${ }^{15}$ The asymmetric and symmetric formations of the $\mathrm{VO}_{4}$ tetrahedron are detected at $c a .327 .8 \mathrm{~cm}^{-1}$ and $362.6 \mathrm{~cm}^{-1}$, respectively. ${ }^{16}$ The peak of $\mathrm{BiVO}_{4}$-OV become weaker and wider than $\mathrm{BiVO}_{4}-\mathrm{P}$, owing to the crystallinity weakening and the lattice distortion, which can manifest some oxygen atoms have been removed from $\mathrm{BiVO}_{4}$, resulting in the formation of oxygen vacancy defects. ${ }^{17}$ This similar phenomenon also appears in the Raman peaks spectrograms that were employed by laser lines 532 and $785 \mathrm{~nm}$ (Fig. S3 and S4†).

According to the $\mathrm{X}$-ray photoelectron spectroscopy of the asprepared $\mathrm{BiVO}_{4}-\mathrm{P}$ and $\mathrm{BiVO}_{4}-\mathrm{OV}$, it seen that indicated the atomic chemical microenvironment on the surface. As we can see high resolution $\mathrm{O}$ 1s spectra in Fig. 2d, the ordinary peak at $529.9 \mathrm{eV}$ for both samples is ascribed to the lattice oxygen ubiquitously in oxide semiconductors. Notably, an additional broad peak at $532.7 \mathrm{eV}$ can be observed, as shown in Fig. 2d, which most probably arises from either oxygen vacancies or adsorbed oxygen species $\left(\mathrm{O}^{-}\right.$and $\left.\mathrm{O}_{2}{ }^{-}\right)$at oxygen vacancies. ${ }^{18}$ They also suggests that oxygen vacancies are stabilized by the adsorbed oxygen species, a typical feature for the defect-rich oxides. Moreover, based on the high resolution $\mathrm{Bi} 4 \mathrm{f}$ spectrum of $\mathrm{BiVO}_{4}$-OV (Fig. S5 $\dagger$ ), the level peaks of $\mathrm{Bi} 4 \mathrm{f}_{7 / 2}$ and $\mathrm{Bi} 4 \mathrm{f}_{5 / 2}$ spin-orbital splitting photoelectrons in the $\mathrm{Bi}^{3+}$ chemical state are at around 161.0 and $155.7 \mathrm{eV}$, respectively. The decreased binding energies of $\mathrm{Bi} 4 \mathrm{f}$ of $\mathrm{BiVO}_{4}-\mathrm{OV}$ are obvious due to the partial reduction of $\mathrm{Bi}^{3+}$ resulting from electrons localized at oxygen vacancy defects. ${ }^{19}$ For comparison with the $\mathrm{BiVO}_{4}-\mathrm{P}$, the $\mathrm{V} 2 \mathrm{p}$ spectrum of the $\mathrm{BiVO}_{4}-\mathrm{OV}$ reveals that the binding energies of $\mathrm{V} 2 \mathrm{p}_{3 / 2}$ and $\mathrm{V} 2 \mathrm{p}_{1 / 2}$ peaks obviously shifted to corresponding to peak position due to the formation of OVs attracting more electrons (Fig. S6†). The similar observation was also reported in other literatures. ${ }^{20}$

To directly prove introduction oxygen vacancies, UV-Vis absorption spectroscopy of the sample was investigated to examine the optical properties of $\mathrm{BiVO}_{4}-\mathrm{P}$ and $\mathrm{BiVO}_{4}-\mathrm{OV}$. As shown in Fig. 2e, this evidence ascertains the imperative roles of OVs during photocatalytic reactions. In intuitive sense, brightyellow and black-yellow separately represent $\mathrm{BiVO}_{4}-\mathrm{P}$ and $\mathrm{BiVO}_{4}$-OV. The formation of oxygen vacancy on $\mathrm{BiVO}_{4}$ surface leads to change color of the sample. Thereby, the better light absorption and accompanied with a reduced band gap, which is reflected by UV-Vis absorption spectra. As shown in Fig. 2e and $\mathrm{f}$, $\mathrm{BiVO}_{4}$-OV certified a much intense continuous absorption over Vis-NIR region. The occurrence of OVs-induced sub-bands excitation from defect states to CB could emerge this appearance, which may cause drastic changes in the optical property. In addition, the absorption edge of $\mathrm{BiVO}_{4}-\mathrm{OV}$ was obvious redshifted, demonstrating the imperative contribution of OVs for band gap narrowing. ${ }^{21}$ Based on the Tauc plot (Fig. 2f), the red slash and blue slash stand for the band gaps of $\mathrm{BiVO}_{4}-\mathrm{OV}$ and $\mathrm{BiVO}_{4}-\mathrm{P}$ at approximated 2.10 and $2.26 \mathrm{eV}$, respectively. The phenomenon of OV-induced band gap narrowing could be ascribed to the down shift of CB minimum. ${ }^{22}$

Photoluminescence (PL) spectroscopy was provided to further ascertain the presence of oxygen vacancy detects. The measurement of PL emission was performed with the excitation at $425 \mathrm{~nm}$ and could reveal the efficiency of charge carries transfer and separation. To understand the fate of photogenerated electrons and holes in semiconductors, Fig. S7† shows the comparison of PL spectra of the $\mathrm{BiVO}_{4}-\mathrm{P}$ and $\mathrm{BiVO}_{4}{ }^{-}$ OV. The both sample PL emission peaks center at about $473 \mathrm{~nm}$. The PL emission intensity of $\mathrm{BiVO}_{4}$ with OVs stay lower than that of $\mathrm{BiVO}_{4}-\mathrm{P}$, which is attributed to the suppressive recombination of electrons and holes, because of the formation of oxygen vacancies. ${ }^{23}$

To elucidate the presence of oxygen vacancy defects for the mechanism of enhanced photocatalytic performance, valence band XPS (VB XPS) analysis was proposed synchronously. It is readily seen from the illustration Fig. 3a, there are two estimated positions at 1.31 and $0.79 \mathrm{eV}$. They represent the $\mathrm{VB}$ maximum energy levels of $\mathrm{BiVO}_{4}-\mathrm{P}$ and $\mathrm{BiVO}_{4}-\mathrm{OV}$, respectively. Combining the above results, corresponding to the $\mathrm{CB}$ minimum was speculated to be downshift, which validate the indispensable contribution of oxygen vacancy for changing the
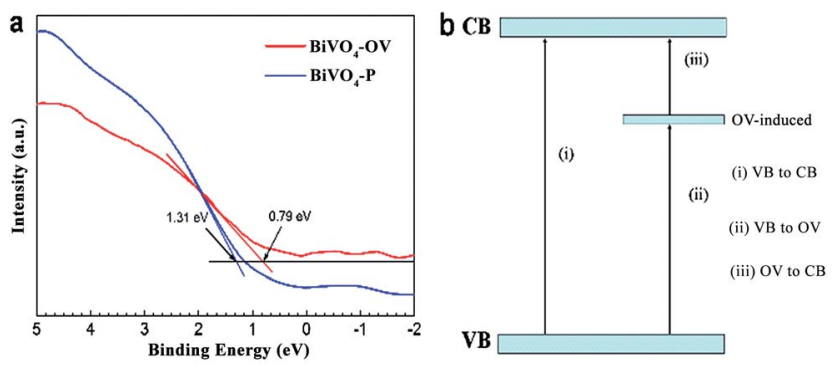

Fig. 3 (a) VB XPS pattern of the $\mathrm{BiVO}_{4}-\mathrm{P}$ and $\mathrm{BiVO}_{4}-\mathrm{OV}$, (b) illustration of $\mathrm{BiVO}_{4}$-OV with possible electrons excitation pathways. 
band edge of the $\mathrm{BiVO}_{4}-\mathrm{OV}$. As delineated in Fig. $3 \mathrm{~b}$, the formation of sub-bands below $\mathrm{CB}$ is attributed to the introduction of OVs. ${ }^{25}$ Fig. $3 \mathrm{~b}$ depicts the energy transfer process, there are three possible procedures to reflect that the electrons could be excited. For instance, (i) electrons transition from VB to $\mathrm{CB}$; (ii) from VB to OV-induced defect states and (iii) from OVinduced defect states to $\mathrm{CB}{ }^{24}$ To look into above situation, the presence of OVs induced defect states serve as springboard for electrons transition, lead to improving the ability of capture more electrons under NIR light irradiation. This novel phenomenon confirms that NIR light could be harvested through the sub-bands excitation from the in-gap bands to $\mathrm{CB}$, due to formation of oxygen vacancies.

The photocatalytic activity of the obtained sample is evaluated by degradation of RhB solution at room temperature and atmospheric pressure under the different irradiation of UV, Vis, NIR light and simulated solar light. The photocatalytic performance experiment was carried through different experimental conditions. As shown in Fig. $4 \mathrm{a}$ and b, the degradation efficiency ratio of $\mathrm{RhB}$ using $\mathrm{BiVO}_{4}-\mathrm{P}$ and $\mathrm{BiVO}_{4}-\mathrm{OV}$ under the UV/ Vis light irradiation for $3 \mathrm{~h}$ reaches the approximate $40 \%$ and $65 \%$, respectively. Upon probing the achieved information, here are several key reasons why $\mathrm{BiVO}_{4}$-OV exhibit higher photocatalytic activity. Firstly, $\mathrm{BiVO}_{4}-\mathrm{OV}$ can absorb more light energy than $\mathrm{BiVO}_{4}-\mathrm{P}$ due to the presence of OVs played an indispensable role to construct new band gap. In addition, the photogenerated electrons that arrived at the surface of $\mathrm{BiVO}_{4}-\mathrm{OV}$ are trapped by the OVs, and then transferred immediately from the catalysts to the adsorbed molecules, thus hindering the photoexcited electron-hole recombination. ${ }^{25}$ Furthermore, the attraction between OVs and organic molecules would result in the formation of unexpected affinity interactions, which lower the energy barrier for interfacial charge transfer. ${ }^{26}$

The previous articles rarely reported that the photocatalytic performance of $\mathrm{BiVO}_{4}$ is response under NIR irradiation. Appreciable degradation rate was detected for $\mathrm{BiVO}_{4}-\mathrm{P}$ (Fig. 4c), which consistent with the theoretical expectation that
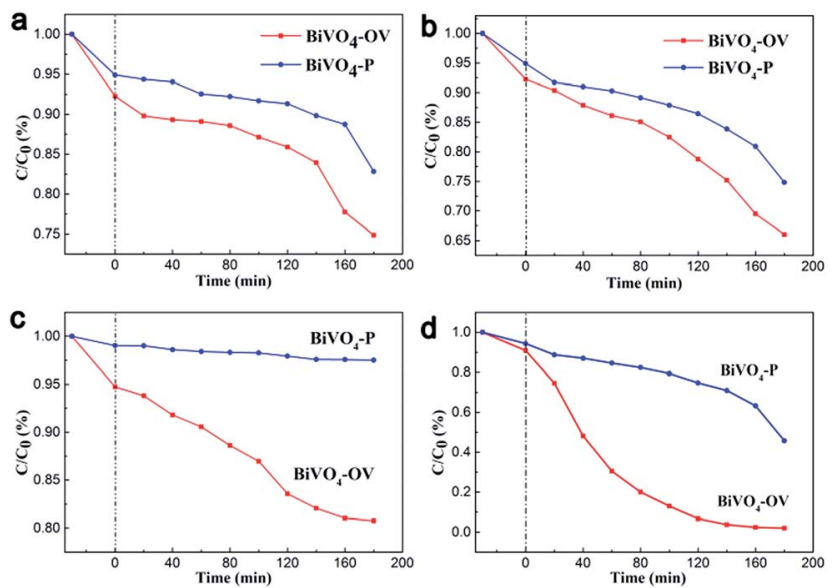

Fig. 4 (a) RhB degradation under UV light illumination (a band-pass filter $\lambda=380 \mathrm{~nm}$ ), (b) Vis light irradiation (a cut off filter $\lambda>420 \mathrm{~nm}$ ), (c) NIR irradiation (a cut off filter $\lambda>800 \mathrm{~nm}$ ) and (d) simulated solar light in the presence of $\mathrm{BiVO}_{4}-\mathrm{P}$ and $\mathrm{BiVO}_{4}$-OV. pristine $\mathrm{BiVO}_{4}$ is not photocatalytic active under NIR light. However, by introduction surface OV detects, the degradation ability of $\mathrm{BiVO}_{4}$ with oxygen vacancy defects was distinctly higher than $\mathrm{BiVO}_{4}-\mathrm{P}$ under NIR irradiation. This demonstrates the presence of $\mathrm{OV}$ defects on the surface of $\mathrm{BiVO}_{4}$ could effectively decompose pollutions to in a noxious productions through gaining low photonic energy NIR light. As aforementioned, surface OVs played a pivotal role to promote the subbands excitation of electrons from OV-induced defect states to CB under long wavelength NIR light. To further examine the photocatalytic capability of $\mathrm{BiVO}_{4}$-OV for converting more energy, RhB degradation experiment was also carried out under simulated solar light irradiation (Fig. 4d). Anticipatorily, $\mathrm{BiVO}_{4}$-OV revealed an excellent light energy conversion, which was distinctly higher than $\mathrm{BiVO}_{4}-\mathrm{P}$ under individual UV, Vis and NIR irradiation.

On account of OVs on $\mathrm{BiVO}_{4}$ surface, capturing low-energy NIR photons could indirectly excite sub-bands. Compared to $\mathrm{BiVO}_{4}-\mathrm{OV}$, the mechanism of organics side chain polyviologen over $\mathrm{BiVO}_{4}-\mathrm{P}$ only allowed direct excitation from VB to CB under UV-Vis light. Based on the analysis summary, the photocatalyst $\mathrm{BiVO}_{4}-\mathrm{OV}$ and $\mathrm{BiVO}_{4}-\mathrm{P}$ process of redox was schematically illustrated in Fig. $5 \mathrm{a}$ and b. Since the preparation of $\mathrm{BiVO}_{4}-\mathrm{OV}$ was employed by the facile solvothermal method and we consider its outstanding photocatalytic performance, it is no doubt that $\mathrm{BiVO}_{4}-\mathrm{OV}$ would be a promising candidate for a wide range of photocatalytic applications over broad spectrum of solar light.

In conclusion, the aforementioned characterization results provide undeniable evidences for the successful formation of surface $\mathrm{OV}$ defects on $\mathrm{BiVO}_{4}-\mathrm{OV}$, rendering prerequisite for studying the roles of OVs play in photocatalytic applications. BiVO $_{4}-\mathrm{OV}$ photoresponse has been successfully attested under UV-Vis-NIR-irradiation, which reflected the presence of OVs. It can not only broaden the activity range of semiconductor oxide from UV to NIR light, but also enhance the carries transformation and effective separation without the incorporation of any noble metal or up-conversion materials. The obtained $\mathrm{BiVO}_{4}$ with the presence of OVs could not only harvest low photonic energy NIR light, simultaneously, it could provide a new train of thought to researchers in this work. Surface defect engineering as important and deep seated insights would maintain a huge promising value of research.
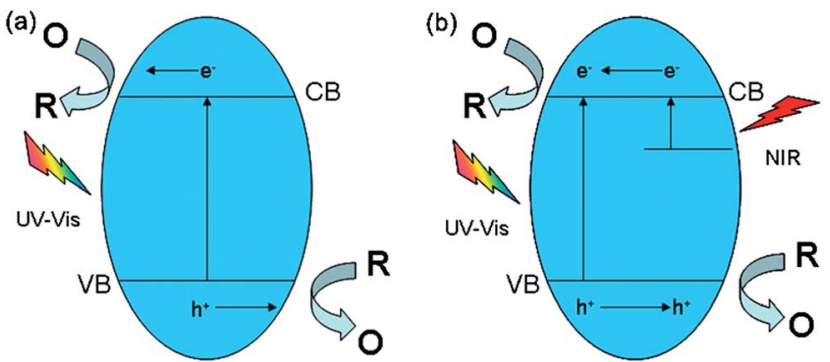

Fig. 5 Schematic diagram for the possible mechanism of occurred redox reaction over (a) $\mathrm{BiVO}_{4}-\mathrm{P}$ and (b) $\mathrm{BiVO}_{4}$-OV. 


\section{Acknowledgements}

This research was supported by the National Natural Science Foundation of China (Grant No. 21476033 and 21577008).

\section{Notes and references}

1 B. Z. Fang, Y. Xing, A. Bonakdarpour, S. C. Zhang and D. P. Wilkinson, ACS Sustainable Chem. Eng., 2015, 3, 2381-2388; Z. Z. Wu, B. Z. Fang, Z. P. Wang, C. L. Wang, Z. H. Liu, F. Y. Liu, W. Wang, A. Alfantazi, D. Z. Wang and D. P. Wilkinson, ACS Catal., 2013, 3, 2101-2107; Z. Xiong, H. B. Wang, N. Y. Xu, H. L. Li, B. Z. Fang, Y. C. Zhao, J. Y. Zhang and C. G. Zheng, Int. J. Hydrogen Energy, 2015, 40, 10049-10062; Y. C. Hao, X. L. Dong, S. R. Zhai, X. Y. Wang, H. C. Ma and X. F. Zhang, Chem. Commun., 2016, 52, 6525.

2 S. Q. Fan, C. Kim, B. Z. Fang, K. X. Liao, G. J. Yang, C. J. Li, J. J. Kim and J. Ko, J. Phys. Chem. C, 2011, 115, 7747-7754; B. Z. Fang, A. Bonakdarpour, K. Reilly, Y. Xing, F. Taghipour and D. P. Wilkinson, ACS Appl. Mater. Interfaces, 2014, 6, 15488-15498; Y. C. Hao, X. L. Dong, S. R. Zhai, X. Y. Wang, H. C. Ma and X. F. Zhang, J. Mater. Chem. A, 2016, 4, 8298.

3 Z. Z. Wu, C. Y. Tang, P. Zhou, Z. H. Liu, Y. S. Xu, D. Z. Wang and B. Z. Fang, J. Mater. Chem. A, 2015, 3, 13050-13056; S. Y. Dong, J. Y. Sun, Y. k. Li, C. F. Yu, Y. H. Li and J. H. Sun, Appl. Catal., B, 2014, 144, 386-393; Y. Zheng, Y. Jiao, Y. H. Zhu, Q. Cai, A. Vasileff, L. H. Li, Y. Han, Y. Chen and S. Z. Qiao, J. Am. Chem. Soc., 2017, 139, 33363339; J. D. Zhang, J. J. Chen, L. P. Xin and M. M. Wang, Mater. Sci. Eng., B, 2014, 179, 6-11.

4 S. B. Gawande and S. R. Thakar, Int. Nano Lett., 2012, 2, 11; S. Fu, Y. D. Liu, Y. Ding, X. Q. Du, F. Y. Song, R. Xiang and B. C. Ma, Chem. Commun., 2014, 50, 2167.

5 H. Tong, S. X. Quyang, Y. P. Bi, N. Umezawa, M. Oshikiri and J. H. Ye, Adv. Mater., 2012, 24, 229.

6 J. Tian, Y. Leng, Z. Zhao, Y. Xia, Y. Sang, P. Hao, J. Zhan, M. Li and H. Liu, Nano Energy, 2015, 11, 419; Y. Sang, Z. Zhao, M. Zhao, P. Hao, Y. Leng and H. Liu, Adv. Mater., 2015, 27, 363; J. Yan, T. Wang, G. Wu, W. Dai, N. Guan, L. Li and J. Gong, Adv. Mater., 2015, 27, 1580.

7 C. Guo, J. Shao, S. Yuan, C. Su, W. Ya, H. Pin, C. Jerome, L. Hong and Y. Guan, Nanoscale, 2015, 7, 3117.

8 I. Justicia, P. Ordejón, G. Canto, J. L. Mozos, J. Fraxedas, G. A. Battiston, R. Gerbasi and A. Figueras, Adv. Funct. Mater., 2002, 14, 1399; X. Pan, M.-Q. Yang, X. Fu, N. Zhang and Y.-J. Xu, Nanoscale, 2013, 5, 3601; J. Wang, Z. Wang, B. Huang, Y. Ma, Y. Liu, X. Qin, X. Zhang and Y. Dai, ACS Appl. Mater. Interfaces, 2012, 4, 4024; M. Guan, C. Xiao, J. Zhang, S. Fan, R. An, Q. Cheng, J. Xie, M. Zhou, B. Ye and Y. Xie, J. Am. Chem. Soc., 2013, 135, 10411.

9 J. Du, J. Qi, D. Wang and Z. Tang, Energy Environ. Sci., 2012, 5, 6914.

10 T. W. Kim and K.-S. Choi, Science, 2014, 343, 990.

11 Y. H. Lv, Y. f. Liu, Y. Y. Zhu and Y. F. Zhu, J. Mater. Chem. A, 2014, 2, 1174.

12 L. F. Sun, Y. Liu, K. Gao, S. Liang, L. Pan and B. Xie, J. Am. Chem. Soc., 2014, 136, 6826.

13 Y. Yu, C. Cao, H. Liu, P. Li, F. Wei, Y. Jiang and W. Song, J. Mater. Chem. A, 2014, 2, 1677.

14 H. Li, J. Shi, K. Zhao and L. Zhang, Nanoscale, 2014, 6, 14168. 15 G. M. Wang, Y. X. Ling, X. H. Lu, F. Qian, Y. X. Tong, J. Z. Zhang, V. Lordi, C. R. Leao and Y. Li, J. Phys. Chem. C, 2013, 117, 10957.

16 S. M. Thalluri, Phys. Chem. Chem. Phys., 2015, 17, 17821.

17 H. Li, J. G. Shi, K. Zhao and L. L. Zhang, Nanoscale, 2014, 6, 14168; Y. H. Lv, Y. F. Liu, Y. Y. Zhu and Y. F. Zhu, J. Mater. Chem. A, 2014, 2, 1174; X. Y. Kong, W. P. Cathie Lee, W. J. Ong, S.-P. Chai and A. R. Mohamed, ChemCatChem, 2016, 8, 1-9.

18 S. P. Berglund, A. J. E. Rettie, S. Hoang and C. B. Mullins, Phys. Chem. Chem. Phys., 2012, 14, 7065.

19 X. Y. Kong, W. P. C. Lee, W.-J. Ong, S.-P. Chai and A. R. Mohamed, ChemCatChem, 2016, 8, 3074.

20 Z. P. Nie, D. K. Ma, G. Y. Fang, W. Chen and S. M. Huang, J. Mater. Chem. A, 2016, 4, 2438.

21 L. Ye, L. Zan, L. Tian, T. Peng and J. Zhang, Chem. Commun., 2011, 47, 6951.

22 G. Zhang, Z. Hu, M. Sun, Y. Liu, L. Liu, H. Liu, C. P. Huang, J. Qu and J. Li, Adv. Funct. Mater., 2015, 25, 3726.

23 X. D. Jiang, Y. P. Zhang, J. Jiang, Y. S Rong, Y. C. Wang, Y. C. Wu and C. X. Pan, J. Phys. Chem. C, 2012, 116, 2261922624; S. Y. Dong, Y. R. Cui, Y. F. Wang, Y. K. Li, L. M. Hu, J. Y. Sun and J. H. Sun, Chem. Eng. J., 2014, 249, 102-110.

24 H. Li, J. Shi, K. Zhao and L. Zhang, Nanoscale, 2014, 6, 14168. 25 W. J. Ong, L. L. Tan, S. P. Chai, S. T. Yong and A. R. Mohamed, Nanoscale, 2014, 6, 1946; W. J. Ong, L. L. Tan, Y. H. Ng, S. T. Yong and S. P. Chai, Chem. Rev., 2016, 116, 7159.

26 K. Xin, C. Yen and C. Siang-Piao, Chem. Commun., 2016, 99, 14242 . 